\title{
Alcohol-induced changes in the developing cerebellum. Ultrastructural and quantitative analysis of neurons in the cerebellar cortex
}

\author{
Eliza Lewandowska, Tomasz Stępień, Teresa Wierzba-Bobrowicz, Paulina Felczak, Grażyna M. Szpak, Elżbieta Pasennik \\ Department of Neuropathology, Institute of Psychiatry and Neurology, Warsaw, Poland
}

\begin{abstract}
Maternal ethanol consumption during pregnancy may cause foetal alcohol syndrome (FAS). Our experiments of ethanoltreated female rats were based on the FAS model in humans; therefore, the results obtained may help explain the clinical mechanism of the disease development. The ultrastructural examination of the cerebellar cortex of ten-day-old rat pups of ethanol-treated dams during pregnancy (group IA), pregnancy and lactation (group IIA), and lactation (group IIIA) revealed that alcohol administration leads to a delayed maturation of Purkinje cells. This was most strongly manifested in the pups of dams treated with ethanol during pregnancy and lactation. Moreover, this study showed degenerative changes in Purkinje cells as well as in granular layer cells in all experimental groups. There was a difference in the ultrastructural picture of both types of dying cells, which might result from different time frame of their sensitivity to ethanol administration. The quantitative analysis showed the most pronounced decrease in the density of Purkinje cells in the posterior superior fissure of cerebellar cortex in the pups of dams treated with ethanol during pregnancy.
\end{abstract}

Key words: foetal alcohol syndrome, Purkinje cells, granular layer cells, ultrastructure, quantitative analysis.

\section{Introduction}

The developing brain is particularly sensitive to the effects of ethanol. They can cause morphological and neurological disorders in the foetus known as foetal alcohol syndrome (FAS). The central nervous system (CNS) dysfunction has inspired the researchers to create an animal model of FAS enabling the detailed studies of neuropathological changes to explain their pathogenesis $[6,32,40]$. Our previous studies have shown that the most widespread neuropathological ethanol-induced changes occur in the cerebellum [39]. The rat cerebellum develops during embryonic and early postnatal phases, and reaches its total maturity in the third month of life [13,21]. Alcohol administration in prenatal and postnatal periods leads to a delayed maturation of the Purkinje cells $[1,36]$ as well as to a significant decrease in the density of both Purkinje and granular cells in the cerebellum $[3,37,38]$. The cerebellar Purkinje cell is one of the largest neurons in the brain and very sensitive to some toxicants, including ethanol [7]. The Purkinje cells in a more advanced state of differentiation are more vulnerable to ethanol-induced cell death than those less matured [4]. It is known that the rat cerebellum is particularly sensitive to ethanol during the first period after birth [19], which is considered to be equivalent to the third trimester of human pre- 
gnancy $[4,10,34]$ - the most critical to ethanol treatment [9]. Factors that disrupt the normal development of the cerebellum can cause different effects, depending on the developmental stage [16], which may be the reason for discrepancies in the results obtained by different researchers in the study of structural and functional ethanol-induced deficits in the cerebellum $[11,18]$.

In our study we have tried to explain how ethanol intoxication affects the ultrastructural picture of the Purkinje cells in ten-day-old rat pups of ethanol-treated dams during pregnancy, pregnancy and lactation, and lactation. In addition, the quantitative evaluation of Purkinje cells was performed and the dying process of Purkinje and granular layer cells was analysed at the ultrastructural level in the same rat pups.

\section{Material and methods}

The investigations were performed on the cerebellum samples derived from ten-day-old pups of Wistar female rats. The experimental groups comprised the pups of females treated with ethanol (12\%, $6 \mathrm{~g} / \mathrm{kg}$ body mass) during pregnancy (group IA), pregnancy and lactation (group IIA) and lactation (group IIIA). The female rats of control groups were treated with isocaloric dextran solution with maltose at the same intervals as those in experimental groups (groups ID, IID and IIID) and given standard diet and water ad libitum in group $C$.

For electron microscopy studies, cerebellum samples were taken from ten-day-old pups of the experimental and control groups. Cerebellar specimens were fixed in $2 \%$ glutaraldehyde solution in cacodylate buffer $\mathrm{pH} 7.4$, and $2 \%$ osmium tetroxide solution in the same buffer. After dehydration in ethanol and propylene oxide, specimens were embedded in Epon 812. Semi-thin sections were stained with toluidine blue to choose appropriate areas. Ultra-thin sections were stained with uranyl acetate and lead citrate and examined with a transmission electron microscope Opton DPS 109 Turbo.

The density of Purkinje cells in the cortex of posterior superior fissure of $9.15 \mathrm{~mm}^{2}$ was analyzed in the experimental and control groups. Preparations were analyzed in Zeiss Axiophot light microscope and then recorded by a set of scanning, including a table with unit labour Märzhäuser LUDL and KS RUN library. STATISTICA 10 was employed to analyze the results obtained. One-way analysis of variance (ANOVA) was used to compare the density of Purkinje cells between experimental and control groups. When ANOVA showed a significant difference, the post hoc Bonferroni and Tukey'a HSD tests were applied to demonstrate the difference.

\section{Results}

In all experimental groups, like in the control group, Purkinje cells formed a single ganglionic layer on the border between granular and molecular layers. Purkinje cells in the control ten-day-old rat pups showed a nearly mature morphology. All their cytoplasm was filled with cellular organelles, especially the mitochondria, and only small regions of cytoplasm contained mainly free ribosomes (Fig. 1). In ten-day-old newborn rats of dams treated with ethanol during pregnancy and/or lactation (groups IA, IIA, IIIA) Purkinje cells were changed to various degrees relative to the control group. They were characterized by large typical nuclei often irregular in outline and irregular distribution of organelles in the cytoplasm of the same cells (Fig. 2A, 2B, $3 A$ ). Usually at one pole, the different size areas of cytoplasm were filled with only numerous free ribosomes and sometimes with single channels of rough endoplasmic reticulum (RER), as well as with single mitochondria, while the remaining cytoplasm was rich in organelles (Fig. 2A, 2B, 3B). The Purkinje cells with area of cytoplasm with accumulation of free ribosomes were most visible in the pups of dams treated with ethanol during pregnancy and lactation (group IIA) and least visible in the pups of dams treated with ethanol during lactation (group IIIA). In some of the Purkinje cells there were areas of cytoplasm containing free ribosomes and numerous channels of rough endoplasmic reticulum in a characteristic arrangement. They were connected to each other giving the impression of the cytoplasm divided into compartments (Fig. 4A, 4B). The system of RER modified so was most frequently observed in Purkinje cells in group IIA, but only occasionally in Purkinje cells in group IIIA. In all the experimental groups we observed Purkinje cells with all or almost all cytoplasm rich in organelles, such as the mitochondria, lysosomes, free ribosomes, Golgi apparatus, channels of rough endoplasmic reticulum, and smooth endoplasmic reticulum (SER) (Fig. 4B, 5A, 5B). In numerous Purkinje cells abundance of mitochondria was seen (Fig. 6A, 6B), in these cells RER channels were usually arranged parallel to each other (Fig. 5A, 5B, 6B). SER channels, usually short, distributed among orga- 
nelles, markedly developed Golgi apparatus and few lamellar bodies with 3-4 compartments were observed (Fig. 4B). In all experimental groups mature Purkinje cells with organelles distributed in all cytoplasm were found (Fig. 6B).

In all experimental groups the ultrastructural changes described above, were accompanied by the degenerative damage of Purkinje cells that varied in intensity. These Purkinje cells were shrunken with markedly increased electron density of the cytoplasm and nucleus characteristic of so-called "dark neuron". They contained the swollen mitochondria, dilated RER channels and cisterns of the Golgi apparatus. In a more advanced stage markedly swollen mitochondria exhibited damaged cristae (Fig. 7A, 7B, 7C).

Dying cells with morphological features of apoptosis were found in the granular layer of cortex cerebellum. They showed the condensed chromatin in both forms of a continuous ring as clumps under the nuclear membrane and dense cytoplasm (Fig. 8A, 8B). Also early and late apoptotic bodies were observed (Fig. 9A, 9B). The majority of cells with apoptotic changes were visible in pups of dams treated with ethanol during pregnancy (group IA) as well as during pregnancy and lactation (group IIA).

In all experimental groups numerous cells at various stages of mitosis occurred in the granular layer of cerebellum (Fig. 10A, 10B).

The results of the quantitative analysis showed significant inter-group differences in the density of Purkinje cells in the posterior superior fissure of cerebellar cortex (Table I). Testing the hypothesis of homogeneity of variance (Levene's test) it was indicated that the assumption of homogeneity of variance was satisfied for ethanol (0.965) and dextran group (0.683). The analysis of variance (ANOVA) for univariate classification revealed statistically significant differences in the density of Purkinje cells in ethanol (0.021) and dextran groups (0.034). Testing the hypothesis of homogeneity of variance (Levene's test) indicated that the assumption of homogeneity of variance was satisfied for group IA (0.150), group IIA (0.675) and group IIIA (0.519) in ethanol groups. The analysis of variance (ANOVA) for univariate classification revealed statistically significant differences (0.028) in the density of Purkinje cells between groups IA and ID (Fig. 11). The post hoc tests (Bonferroni and Tukey HSD tests) showed statistically significant differences $(p<0.05)$ between groups IA and IIA (0.013), and between groups IA and IIIA (0.001).

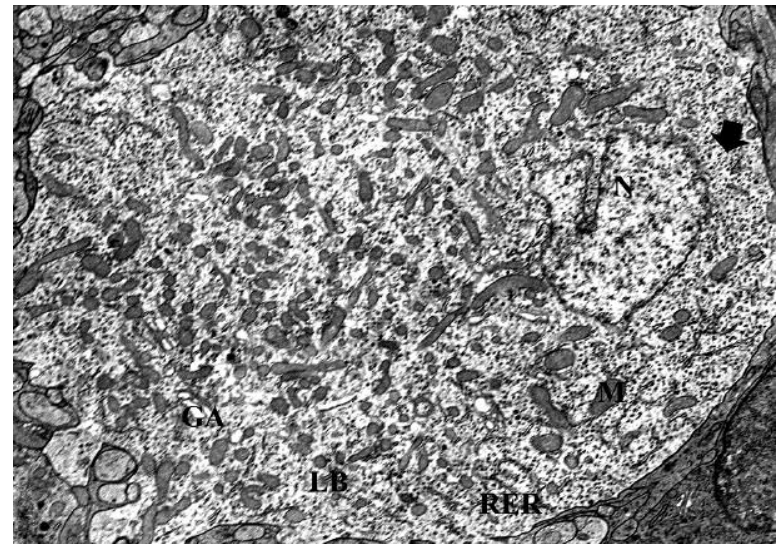

Fig. 1. Control group. Mature Purkinje cell. $\mathrm{N}$ - nucleus, $\mathrm{M}$ - mitochondria, RER - rough endoplasmic reticulum, GA - Golgi apparatus, LB - lamellar bodies, (arrow) - ribosomes. Orig. magn. $\times 3000$.

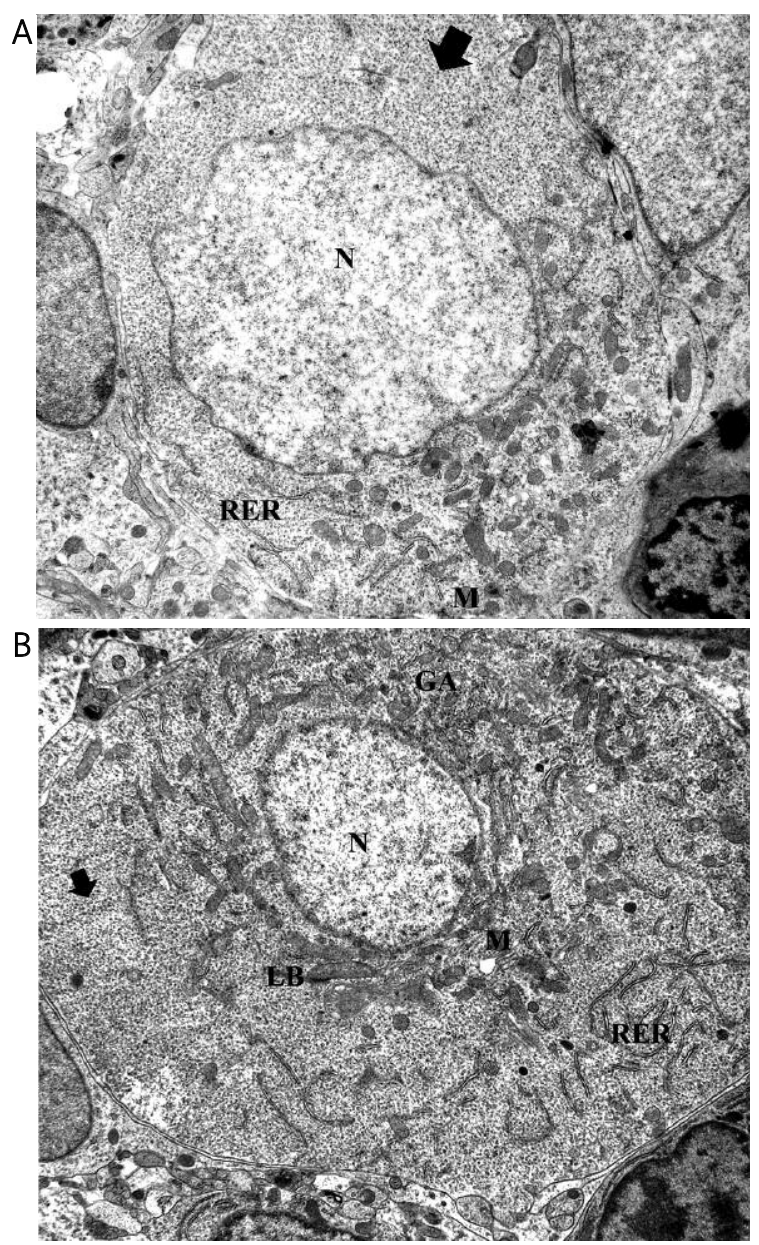

Fig. 2. A, B) Experimental group IIA. Purkinje cells with big areas filled with free ribosomes (arrows). $\mathrm{N}$ - nucleus, $\mathrm{M}$ - mitochondria, RER - rough endoplasmic reticulum, GA - Golgi apparatus, LB - lamellar bodies. Orig. magn. $\times 3000$. 

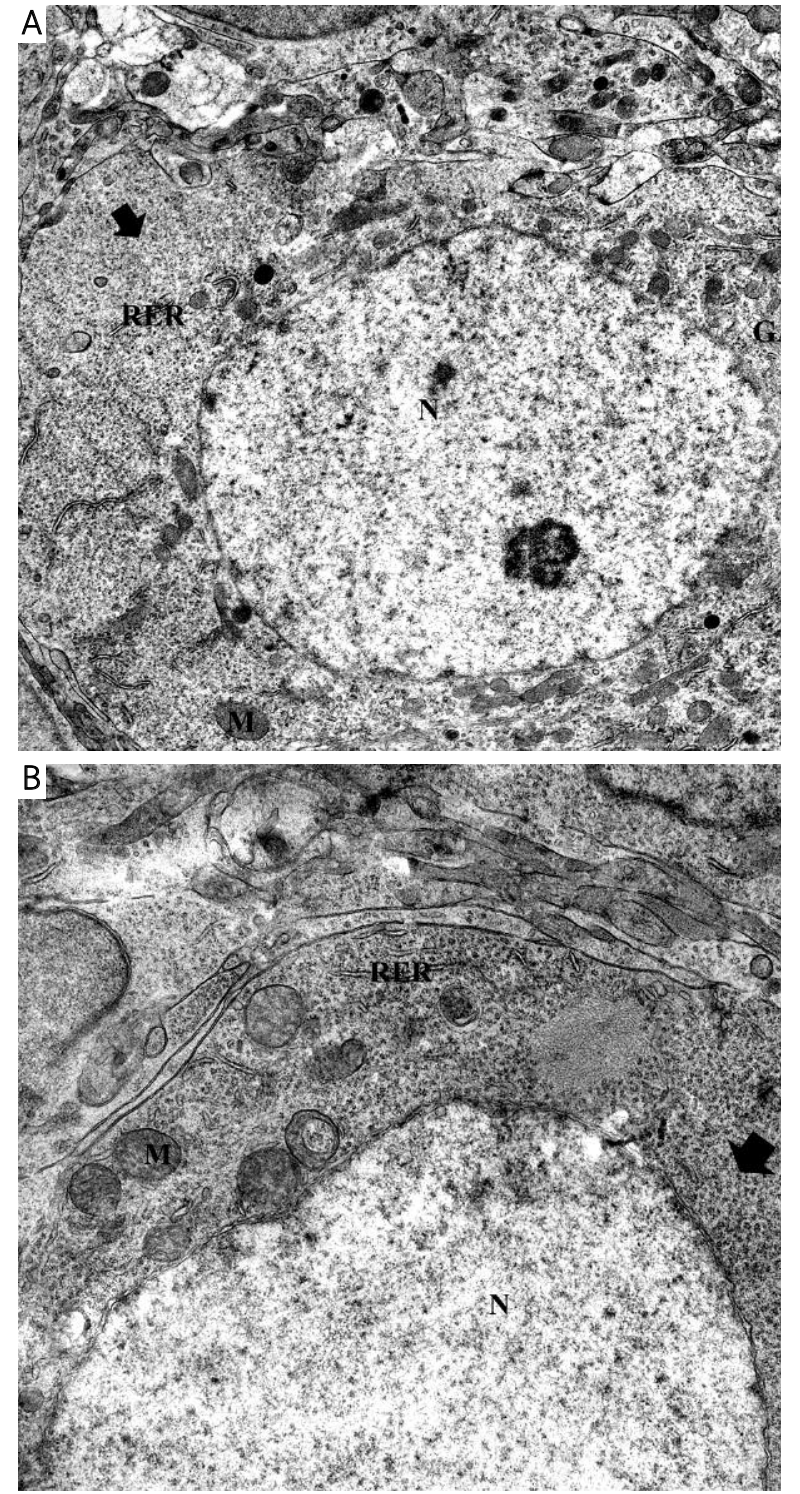

Fig. 3. A, B) Experimental group IA. Purkinje cells with areas filled with free ribosomes (arrows) and single channels of RER - rough endoplasmic reticulum and mitochondria (M). $\mathrm{N}$ - nucleus, GA - Golgi apparatus. A) Orig. magn. $\times 4400$; B) Orig. magn. $\times 7000$.

\section{Discussion}

It is commonly known that the toxic effect of ethanol consumption during pregnancy causes different pathological changes in the developing foetus, including degenerative changes in the cerebellum observed in children with FAS and in the animal model.
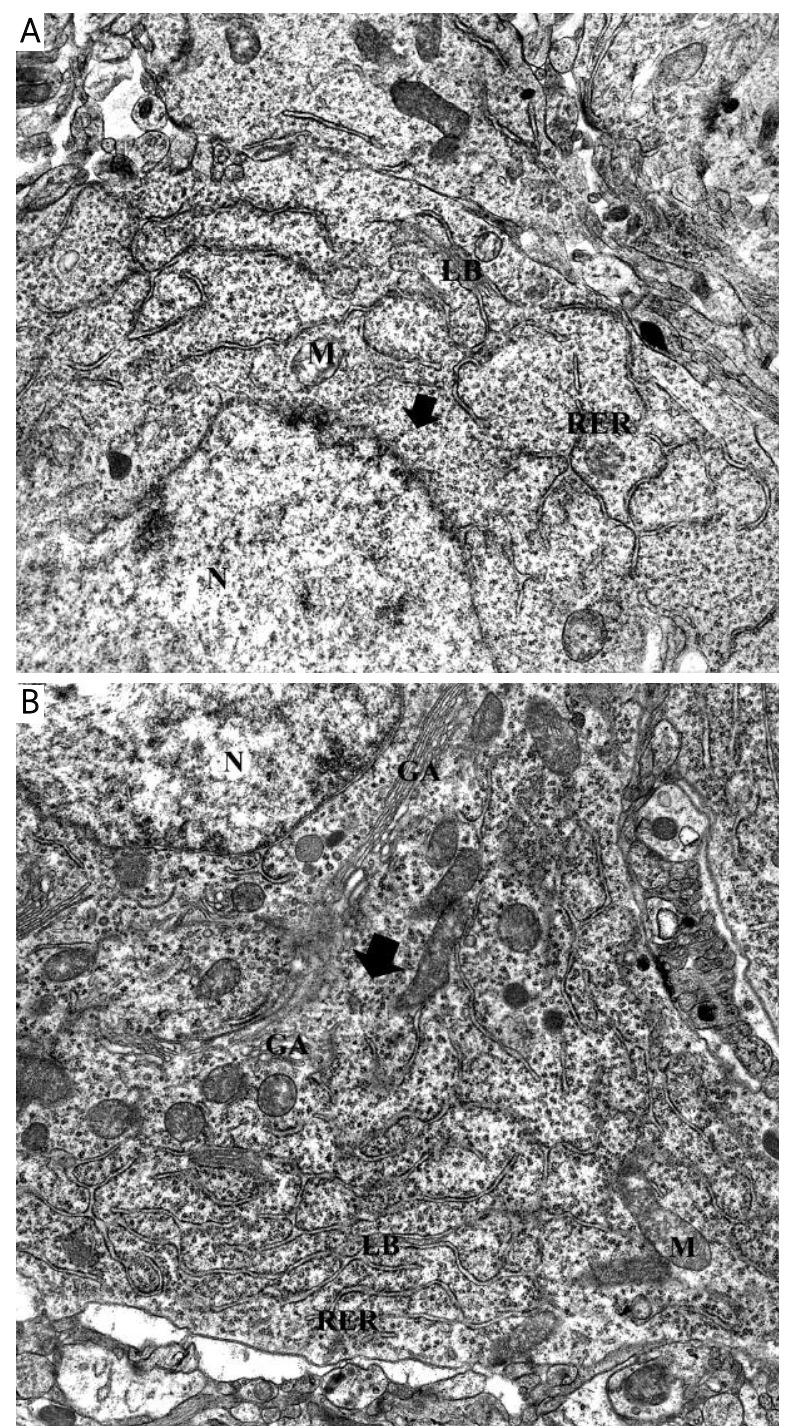

Fig. 4. A, B) Experimental group IA. A) Purkinje cell with numerous branched channels of RER, and single mitochondria $(M)$ into the area filled with free ribosomes (arrow). LB - lamellar bodies. B) In the vicinity of nucleus prominent Golgi apparatus (GA). RER - rough endoplasmic reticulum, free ribosomes (arrow), $\mathrm{N}-$ nucleus. Orig. magn. $\times 7000$.

Our ultrastructural examination of cerebellar cortex revealed that the major alterations concerned disorders of morphology of Purkinje cells and their degeneration, as well as the degeneration of granular layer cells in all experimental groups. It should be noted that such cellular changes, although a few, were also present in the control groups. 


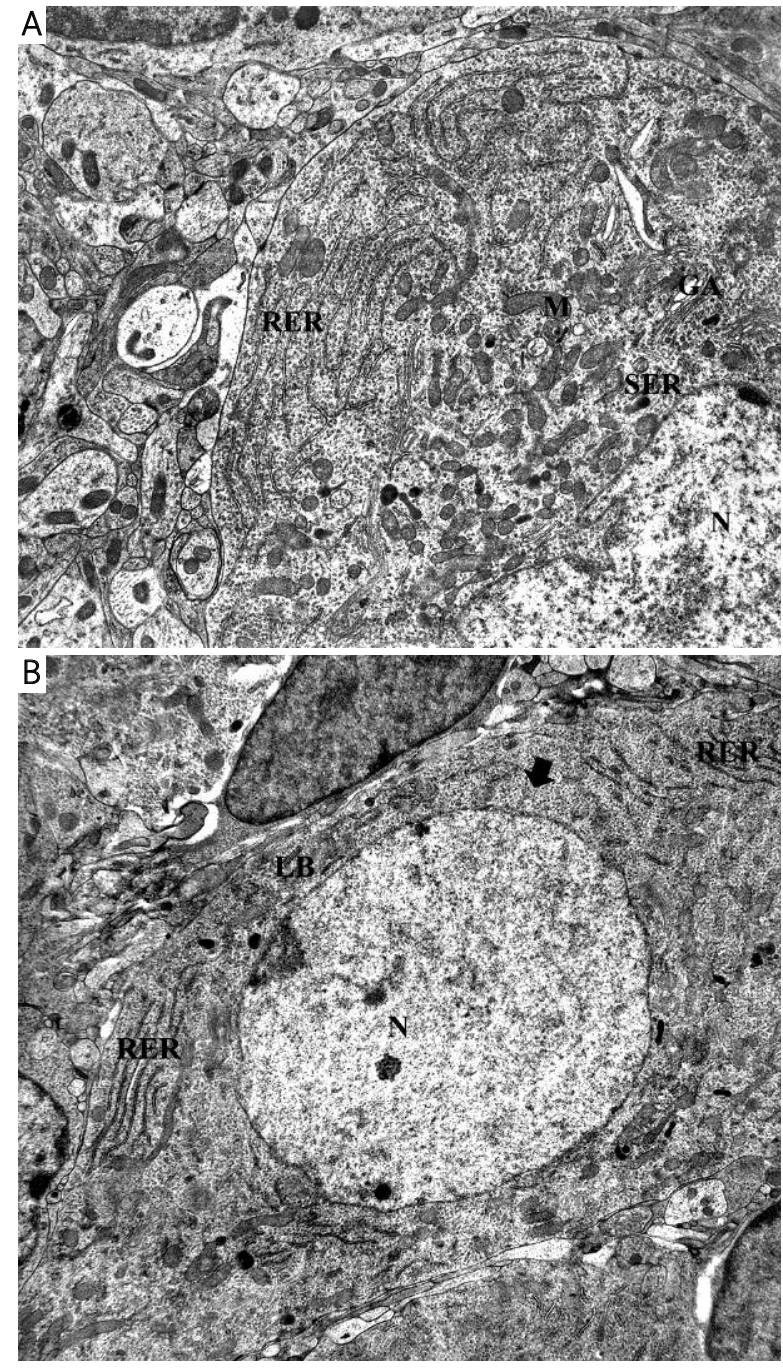

Fig. 5. A) Experimental group IA. B) Experimental group IIA. Purkinje cells displaying numerous RER channels arranged parallel to each other under plasma membrane. LB - lamellar bodies, $\mathrm{M}$ - mitochondria, N - nucleus, (arrow) - ribosomes, GA - Golgi apparatus, SER - smooth endoplasmic reticulum. A) Orig. magn. $\times 4400$; B) Orig. magn. $\times 3000$.

Only few data on ultrastructural changes in the Purkinje cells of foetal and neonatal rats induced by ethanol intoxication exist in the literature [14,36,37].

In the cerebellar cortex of offsprings of alcohol drinking dams, both before mating and during pregnancy, demonstrated delayed maturation of Purkinje cells $[35,36]$, which may be caused by undernutrition [29].

Similarly, in our study of the cerebellar cortex of tenday-old rat pups of ethanol-treated dams the major
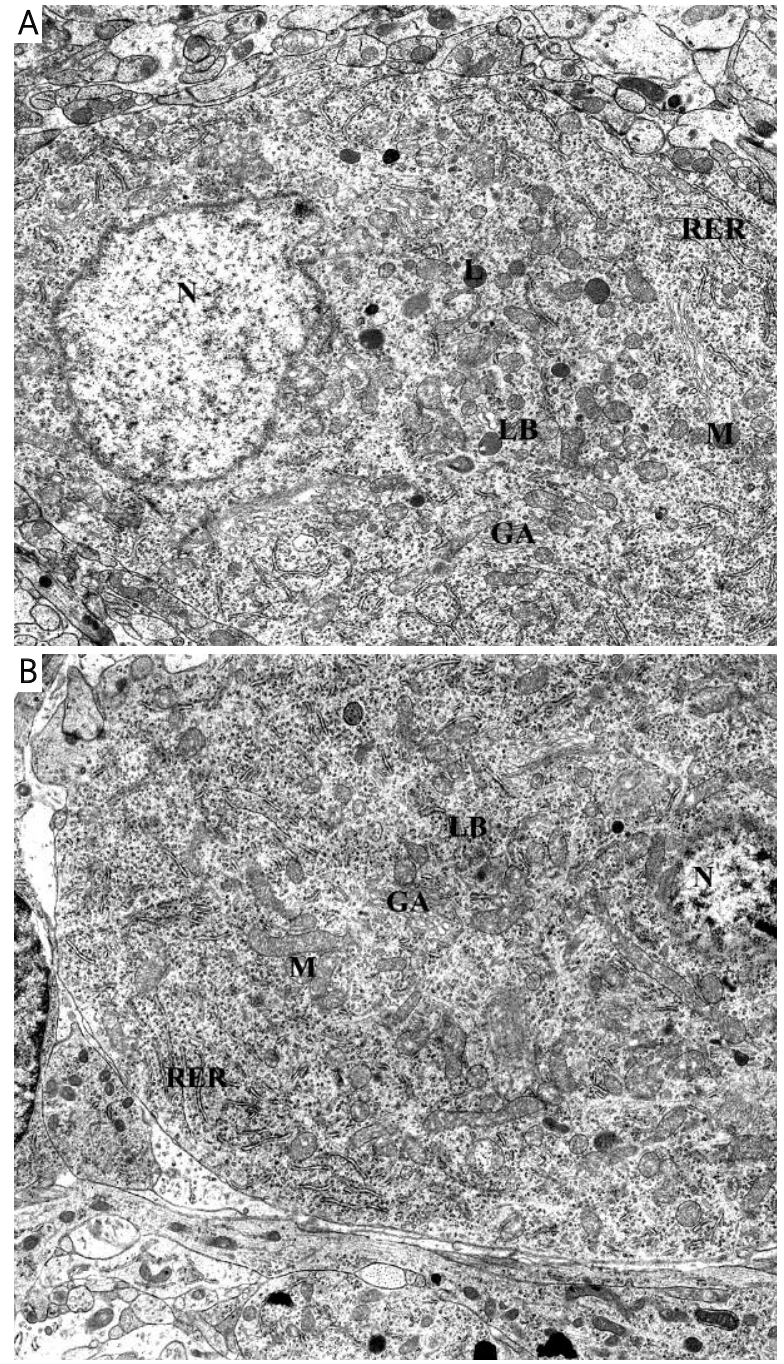

Fig. 6. A, B) Experimental group IA. Cytoplasm of Purkinje cells rich in organelles: $M$ - mitochondria, N - nucleus, RER - rough endoplasmic reticulum, GA - Golgi apparatus, LB - lamellar bodies, $L-$ lysosomes. Orig. magn. $\times 4400$.

ultrastructural change within the perikarya of Purkinje cells was the presence of cytoplasm region filled with free ribosomes only. These areas of cytoplasm varied in size and were the most numerous in the pups of dams treated with ethanol during pregnancy and lactation (group IIA). In the remaining cytoplasm of Purkinje cell organelles, such as mitochondria, vesicles, Golgi apparatus and endoplasmic reticulum were mostly located. In the majority of Purkinje cells abun- 


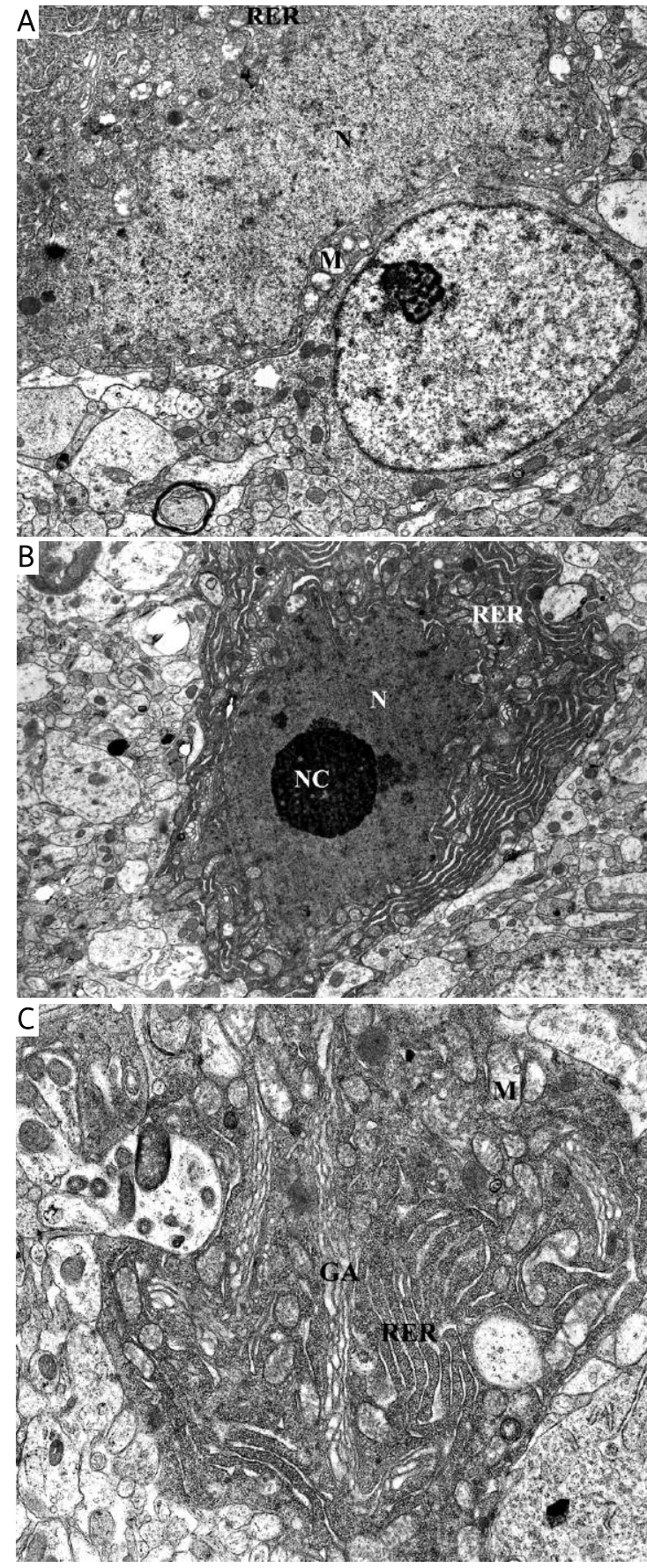

Fig. 7. A, B, C. A) Experimental group IIA. Purkinje cell showing early changes of "dark neuron". $\mathrm{N}$ - nucleus, $\mathrm{M}$ - swollen mitochondria, RER dilated channels. B) Experimental group IIA. Purkinje cell with a considerably increased density of the cytoplasm and nucleus (N). RER - dilated channels, NC - nucleolus. C) Experimental group IIIA. Dark cytoplasm of Purkinje cell filled with distinctly dilated channels of RER, cisterns of the Golgi apparatus (GA) and swollen mitochondria (M). A, C) Orig. magn. $\times 4400$; B) Orig. magn. $\times 3000$. dant mitochondria were seen. Interestingly, in the region of cytoplasm filled with free ribosomes, short RER channels appeared first. This was followed by the accumulation of numerous branched RER channels. The arrangement of RER channels, accompanied by a few mitochondria, gave an impression of compartmentalization of cytoplasm in this region. In our understanding, the first stage of maturation of Purkinje cells cytoplasm in this area was manifested by RER expansion. The organelles, such as mitochondria, Golgi apparatus, dense bodies resembling lysosomes, smooth endoplasmic reticulum channels and others appeared in this area during the final stage of maturation. In mature Purkinje cells, all perikarya were filled with organelles listed above, but the arrangement and distribution of RER channels were changed. They were arranged parallel to one another, while SER channels were short and single. As evidenced endoplasmic reticulum plays a major role in detoxification.

Ultrastructural changes in endoplasmatic reticulum of Purkinje cells may influence ethanol metabolism by cytochrome P450IIEl. Alcohol can also be oxidized to acetaldehyde by CYP2E1 that generates hydrogen peroxide [12]. The production of free radicals derived from hydrogen peroxide leads to oxidative stress and cell death. Free radicals are highly reactive to all the molecular targets: lipids, proteins and nucleic acids, modifying their chemical structure and generating oxidation-derived products [23].

To our best knowledge, in the literature there is no description of the above mentioned changes in the maturing region of cytoplasm of Purkinje cells. Only Volk et al. [36] believe that a delayed cytoplasmic maturation of Purkinje cells mainly involves the rough endoplasmatic reticulum. Biochemical studies have shown the decrease in ribosomal protein biosynthesis in the rat brain, as well as in total RNA and DNA content and disaggregation of polysomes into free ribosomes of neonates suckling on alcohol-fed mothers $[15,22,24,26,27,28]$. We believe that the delayed maturation of Purkinje cells may be associated with a decreased ribosomal protein biosynthesis. On the other hand, in the maturing Purkinje cells accumulation of free ribosomes in basal parts of cytoplasm was observed. However, maternal ethanol consumption induces alterations in the proprieties of brain ribosomes and RNA metabolism, which may affect the capacity of protein synthesis in neural cells in the foetal and neonatal rat brain [26]. At the first stage of maturation, an increasing number of membrane-bound ribo- 

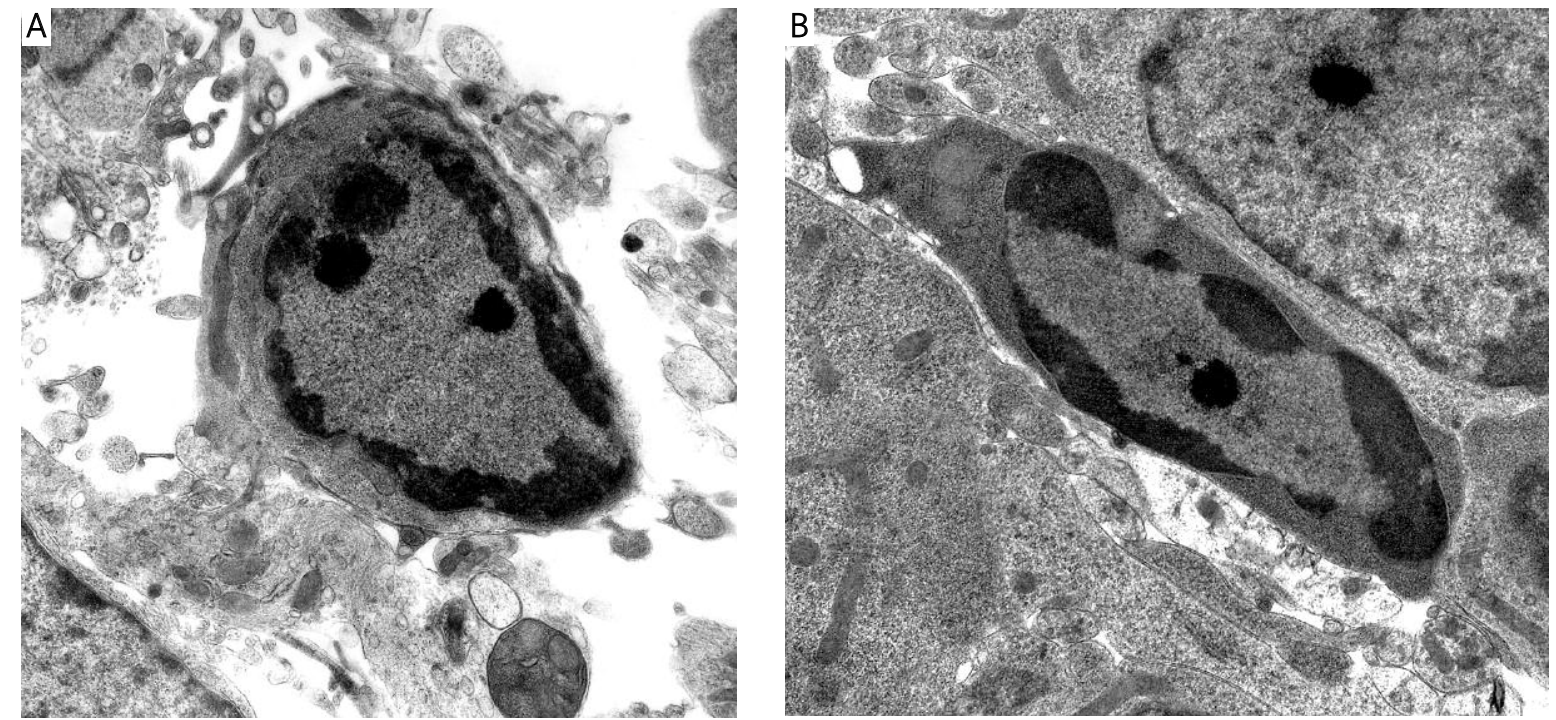

Fig. 8. A, B) Experimental group IIA. Granular layer cells showing apoptotic-like morphology. Orig. magn. $\times 4400$.
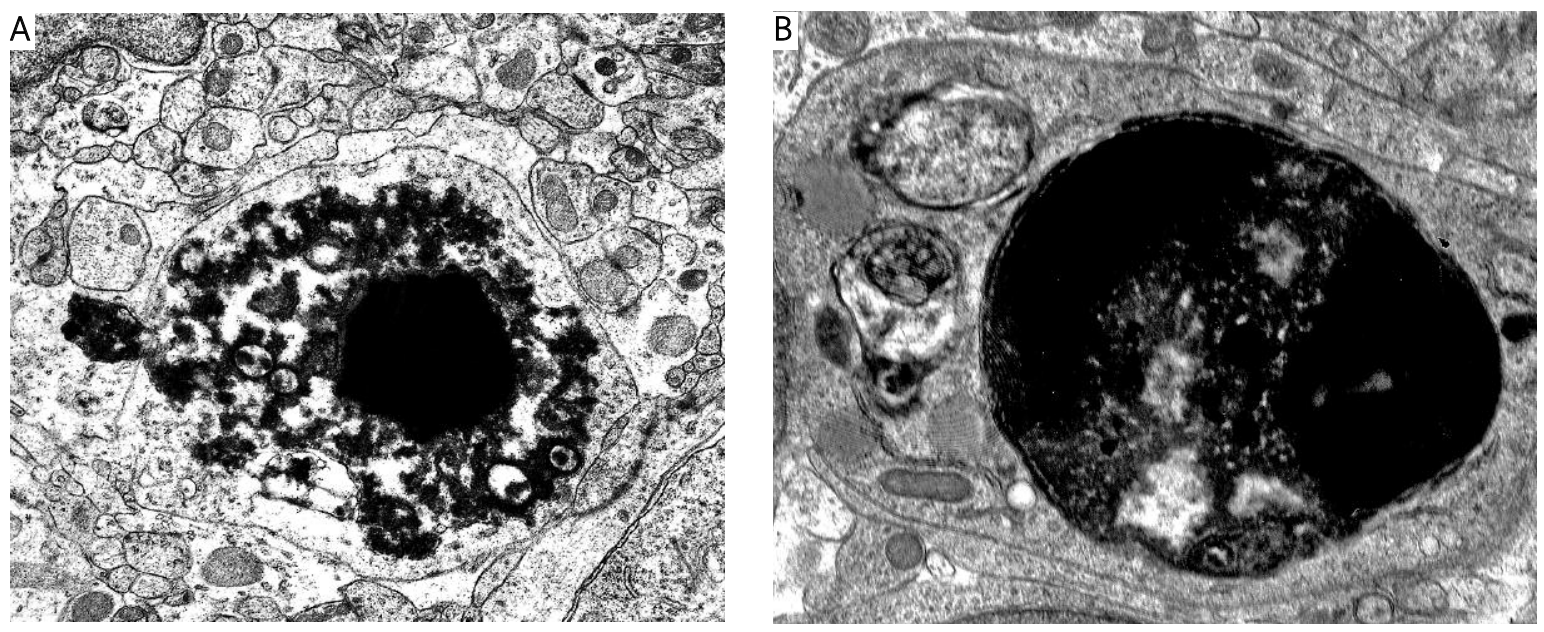

Fig. 9. A, B) Experimental group IA. A) Early apoptotic body. B) Late apoptotic body. A) Orig. magn. ×4400,

B) Orig. magn. $\times 7000$.
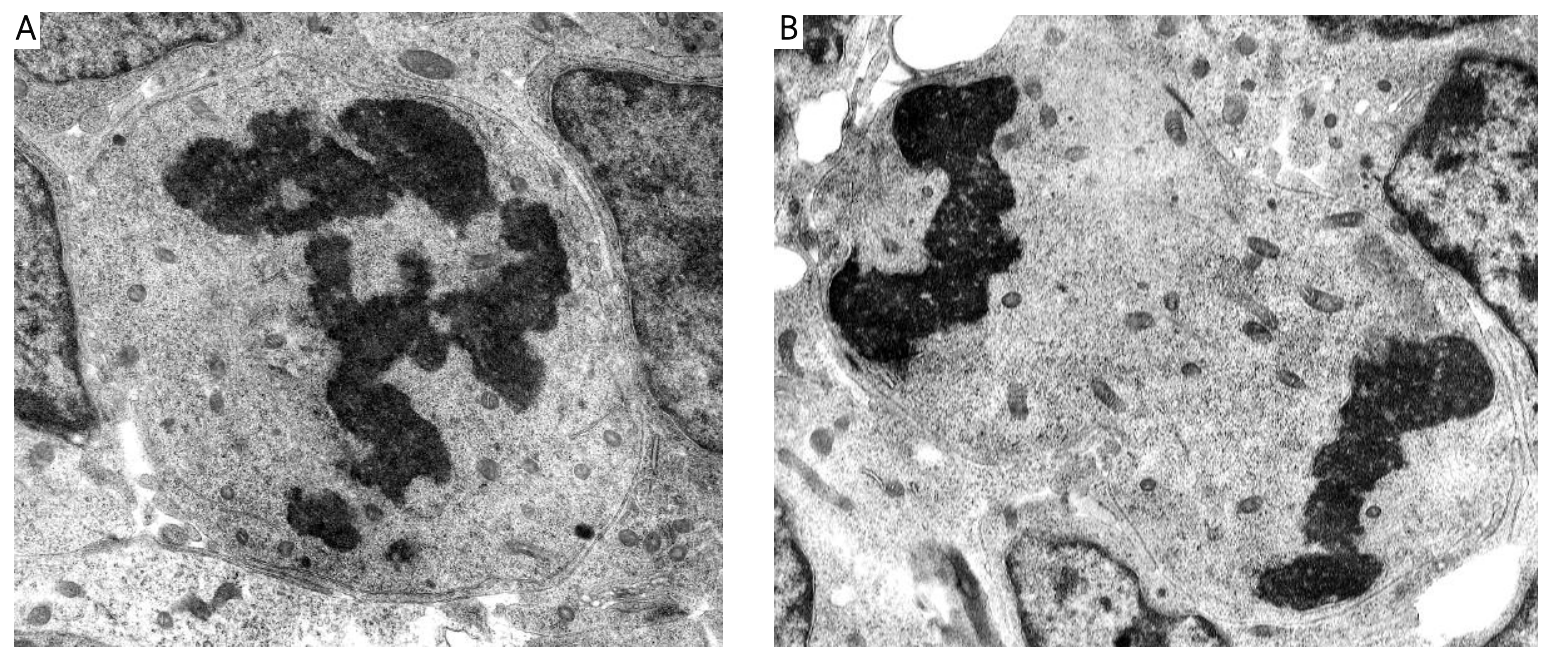

Fig. 10. A, B) Experimental group IA. Mitotic cells in granular layer. Orig. magn. $\times 4400$. 
Table I. Average density of Purkinje cells in cerebellar cortex in the experimental and control groups

\begin{tabular}{|ccccc|}
\hline Group & Solution administered & Duration of treatment & Number of cases & Density of Purkinje cells \\
\hline \multicolumn{5}{|c|}{ Experimental groups } \\
\hline IA & Ethanol & Pregnancy & 7 & $19.75 \pm 6.64$ \\
\hline IIA & Ethanol & Pregnancy and lactation & 8 & $23.99 \pm 7.15$ \\
\hline IIIA & Ethanol & Lactation & 7 & $25.01 \pm 8.05$ \\
\hline & & Control groups & \\
\hline ID & Dextran & Pregnancy & 7 & $23.31 \pm 6.04$ \\
\hline IID & Dextran & Pregnancy and lactation & 6 & $26.39 \pm 7.49$ \\
\hline IIID & Dextran & Lactation & 7 & $27.01 \pm 8.47$ \\
\hline C & Not treated & - & 8 & $26.53 \pm 8.17$ \\
\hline
\end{tabular}

somes and few mitochondria are found among numerous free ribosomes. The mechanisms which lead to the maturation of Purkinje cells remain unclear. We observed proliferation of mitochondria in Purkinje cells. It is generally known that ethanol induces triacylglycerols metabolism in mitochondria [33].

In addition to the above-mentioned changes, some of Purkinje and granular layer cells revealed morphological features of dying cells in all experimental groups. The number of degenerating cells in the granular layer was much lower. It is interesting to note that the ultrastructural picture of both types of dying cells was different, which might result from the difference in the time frame of sensitivity to ethanol administration between Purkinje and granular cells [33]. Although it is known that exposure to ethanol during the development of cerebellum may lead to loss of Purkinje and

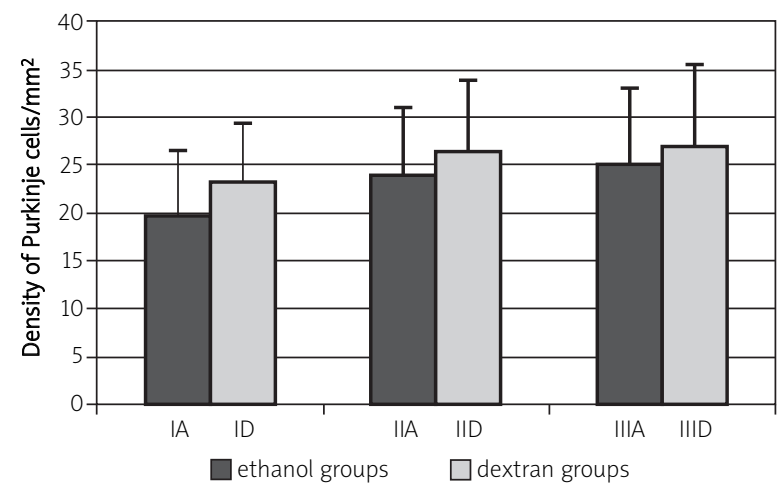

Fig. 11. Average density of Purkinje cells in posterior superior fissure of cerebellar cortex. granular cells $[8,10,18,34]$ however, the mechanism of cellular death still remains unclear [5,42]. Based on the immunohistochemical and ultrastructural studies some authors consider that ethanol-induced degeneration of Purkinje cells involves an apoptotic mechanism [5,18]. On the other hand, Lee et al. [17] based on the same research techniques conclude that neither alcohol apoptosis nor necrosis is the response to death of Purkinje cells. The degenerating Purkinje cells appeared as "dark" ischemic neurons observed in various pathological processes and the action toxins [31,41]. According to some authors, ultrastructural picture of these cells may be responsible for some phase of apoptosis [25] or is caused by improper procedures of material preparation [13]. In our ultrastructural examination, the Purkinje cells differed from typical apoptotic cells. They had no chromatin fragmentation, which is a characteristic feature of apoptosis. Moreover, apoptotic bodies were absent in Purkinje cells layer. Our study showed degenerating shrunken and dark like Purkinje cells in all experimental groups after ethanol intoxication in the developing cerebellum. They were like Purkinje cells in experimental encephalopathy induced by chronic application of valproate [30]. So-called "dark neurons" showed features of aponecrosis [31], while dying cells of the granular layer displayed typical morphological features of apoptosis, like condensation of chromatin along nuclear membrane, dense clumps and shrinkage of nuclei and cytoplasm. The presence of apoptotic bodies in this layer provides additional evidence of apoptosis. An interesting question is why different mechanisms are involved in the death of the two types 
of neurons in response to alcohol. In the light of current data, this issue remains unanswered. So is the question on whether death is a direct result of the toxic effects of ethanol or a delayed secondary response to an earlier death of Purkinje cells or by later maturation of granular cells [5].

The quantitative analysis also showed the decrease in the density of Purkinje cells. The most pronounced decrease in their density was observed in the posterior superior fissure of cerebellar cortex in the pups of dams treated with ethanol during pregnancy (group IA) and the least pronounced in the pups of dams treated with ethanol during lactation (group IIIA). We showed statistically significant differences between groups IA and IIA, and groups IA and IIIA. In our study, the highest sensitivity to ethanol treatment was not observed in mature Purkinje cells, but in their differential phase of thr postnatal period. However, it has been reported that Purkinje cells in a more advanced state of differentiation are more vulnerable to alcoholinduced cell death [3,4]. Marcusen et al. [20] have shown that the developing Purkinje cells are more vulnerable to alcohol-induced depletion during differentiation than during neurogenesis. After ethanol exposure Hamre and West [10] noted the greatest loss of Purkinje cells on postnatal days 4 to 5 . Some differences in the density of Purkinje cells in pups of dams treated with ethanol may arise from different approaches to the model of ethanol treatment. Some FAS models indicate about $20 \%$ decrease in the density of Purkinje cells [2,34], but Volk et al. [36] showed no difference in the number of Purkinje cells. The loss of Purkinje cells was observed after both single ethanol exposure during the postnatal period $[8,18,34]$. Alcohol consumption during pregnancy has a significant effect on the human foetus. Our experiment of ethanol treatment in rats based on the FAS model in humans and our findings bring us closer to the explanation of the clinical mechanism by which this disease develops.

To sum up, ethanol leads to a delayed maturation of Purkinje cells. We observed a lot of immature Purkinje cells in pups of dams treated with ethanol during pregnancy and lactation. However, we also showed the decrease in the density of Purkinje cells in the posterior superior fissure of cerebellar cortex in pups of dams treated with ethanol during pregnancy. The difference in the ultrastructural picture of dying Purkinje cells and cells of granular layer was evidenced and may depend on the stage of cellular development.

\section{References}

1. Abel EL. Consumption of alcohol during pregnancy: A review of effects on growth and development of offspring. Hum Biol 1982; 54: 421-453.

2. Backman C, West JR, Mahoney JC, Palmer MR. Electrophysiological characterization of cerebellar neurons from adult rats exposed to ethanol during development. Alcohol Clin Exp Res 1998; 22: 1137-1145.

3. Bauer-Moffet C, Altman J. The effect of ethanol chronically administered to preweanling rats on cerebellar development: a morphological study. Brain Res 1977; 119: 249-268.

4. Bonthius DJ, West JR. Alcohol-induced neuronal loss in developing rats: increased brain damage with binge exposure. Alcohol Clin Exp Res 1990; 14: 107-118.

5. Dikranian K, Qin YQ, Labruyere J, Nemmers B, Olney JW. Ethanolinduced neuroapoptosis in the developing rodent cerebellum and related brain stem structures. Developmental Brain Research 2005; 155: 1-13.

6. Driscoll CD, Streissguth AP, Riley EP. Prenatal ethanol exposure: comparability of effects in humans and animal models. Neurotoxicol Teratol 1990; 12: 231-237.

7. Fonnum F, Lock EA. Cerebellum as a target for toxic substances. Toxicol Lett 2000; 112: 9-16.

8. Goodlett CR, Eilers AT. Alcohol-induced Purkinje cell loss with a single binge exposure in neonatal rats: a stereological study of temporal windows of vulnerability. Alcohol Clin Exp Res 1997; 21: 738-744.

9. Guerri C. Neuroanatomical and neurophysiological mechanisms involved in central nervous system dysfunctions induced by prenatal alcohol exposure. Alcohol Clin Exp Res 1998; 22: 304-312.

10. Hamre KM, West JR. The effects of the timing of ethanol exposure during the brain growth spurt on the number of cerebellar Purkinje and granule cell nuclear profiles. Alcohol Clin Exp Res 1993; 17: 610-622.

11. Jaatinen P, Rintala J. Mechanisms of ethanol-induced degeneration in the developing, mature, and aging cerebellum. Cerebellum 2008; 7: 332-347.

12. Ji C. Mechanisms of alcohol-induced endoplasmic reticulum stress and organ injuries. Biochem Res Int 2012; 2012: 216450. Epub 2011 Oct 26.

13. Jortner BS. The return of the dark neuron. A histological artifact complicating contemporary neurotoxicologic evaluation. Neurotoxicology 2006; 27:628-634.

14. Köksal M, Ilgaz C, Erdogan D, Özo ul C, Tong EK, Kalender H. Ultrastructure of rat pup's Purkinje neurons whose mothers were exposed to ethanol during pregnancy and lactation. Intem J Neuroscience 2005; 115: 1669-1686.

15. Lang CH, Wu D, Frost RA, Jefferson LS, Vary TC, Kimball SR. Chronic alcohol feeding impairs hepatic translation initiation by modulating elF2 and elF4E. Am J Physiol 1999; 277: 805-814.

16. Laure-Kamionowska M, Maślińska D. Calbindin positive Purkinje cells in the pathology of human cerebellum occurring at the time of its development. Folia Neuropathol 2009; 47: 300-305.

17. Lee Y, Park DB, Song JH, Kang HK, Kang JH, Maier SE, West JR. Death mechanisms of cerebellar Purkinje cells induced by ethanol treatment in developing Pd5 Rat. 2002; Program No.823.5. Abstract Viewer/Itinerary Planner, Society for Neuroscience, Washington, DC. 
18. Light KE, Belcher SM, Pierce DR. Time course and manner of Purk inje neuron death following a single ethanol exposure on postnatal day 4 in the developing rat. Neuroscience 2002; 114: 327-337.

19. Luo J. Mechanisms of ethanol-induced death of cerebellar granule cells. Cerebellum 2012; 11: 145-154.

20. Marcussen BL, Goodlett CR, Mahoney JC, West JR. Developing rat Purkinje cells are more vulnerable to alcohol-induced depletion during differentiation than during neurogenesis. Alcohol 1994; 11 147-156.

21. McKay BE, Turner RW. Physiological and morphological development of the rat cerebellar Purkinje cell. J Physiol 2005; 567: 829-850.

22. Oratz M, Rothschild MA, Schreiber SS. Alcohol amino acids, and albumin synthesis. III. Effects of ethanol, acetaldehyde, and 4-methylpyrazole. Gastroenterology 1978; 74: 672-676.

23. Pradeep H, Diya JB, Shashikumar S, Rajanikant GK. Oxidative stress - assassin behind the ischemic stroke. Folia Neuropathol 2012; 50: 219-230.

24. Preedy VR, Peters TJ. An investigation into the effects of chronic ethanol feeding on hepatic mixed protein synthesis in immature and mature rats. Alcohol Alcohol 1989; 24: 311-318.

25. Ratan RR, Murphy TH, Baraban JM. Oxidative stress induces apoptosis in embryonic cortial neurons. J Neurochem 1994; 62: 376-379.

26. Rawat AK. Ribosomal protein synthesis in the fetal and neonatal rat brain as influenced by maternal ethanol consumption. Res Commun Chem Pathol 1975; 12: 723-732.

27. Rawat AK. Effects maternal ethanol consumption on fetal and neonatal rat hepatic protein synthesis. Biochem J 1976; 160: 653-661.

28. Rothschild MA, Oratz M, Morland J, Schreiber SS, Burks A, Martin B. Effects of ethanol on protein synthesis and secretion. Pharmacol Biochem Behav 1980; 13: 31-36.

29. Sima A. Persson $L$. The effect of pre- and postnatal undernutrition on the development of the rat cerebellar cortex. Neurobiology 1975; 5: 23-34.

30. Sobaniec-Łotowska ME. Ultrastructure of Purkinje cell perikarya and their dendritic processes in the rat cerebellar cortex in experimental encephalopathy induced by chronic application of valproate. Int J Exp Path 2001; 82: 337-348.

31. Sobaniec-Łotowska ME, Łotowska JM. The neuroprotective effect of topiramate on the ultrastructure of pyramidal neurons of the hippocampal CA1 and CA3 sectors in an experimental model of febrile seizures in rats. Folia Neuropathol 2011; 49: 230-236.

32. Stojanović N, Budec M, Jovcić G, Bugarski , Teodorović V. Effect of a single dose of ethanol on granulopoiesis in female rats; relationship to phase of estrous cycle. J Stud Alcohol 1996; 57: 344 348.

33. Taranukhin AG, Taranukhina EY, Saransaari P, Podkletnova IM, Pelto-Huikko M, Oja SS. Neuroprotection by taurine in ethanol-induced apoptosis in the developing cerebellum. J Biomed Sci 2010; 24 (Suppl 1): 12.

34. Thomas J. Goodlett Ch.R, West J. Alcohol-induced Purkinje cell loss depends on developmental timing of alcohol exposure and correlates with motor performance. Dev Brain Res 1998; 105: 159-166.

35. Volk B. Delayed cerebellar histogenesis in embryo-fetal alcohol syndrome. Light microscopic study of the rat cerebellum. Acta Neuropathol 1977; 39: 157-163.
36. Volk B, Maletz J, Tiedemann M, Mall G, Klein C, Berlet HH. Impaired maturation of Purkinje cells in the fetal alcohol syndrome of the rat. Acta Neuropathol 1981; 54: 19-29.

37. Volk B. Cerebellar histogenesis and synaptic maturation following pre- and postnatal alcohol administration. Acta Neuropathol 1984; 63: 57-65.

38. West JR, Goodlett CR, Bonthius DJ, Hamre KM, Marcussen BL Cell population depletion associated with fetal alcohol brain damage: Mechanisms of BAC-dependent cell loss. Alcohol Clin Exp Res 1990; 14: 813-818.

39. West JR. Acute and long-term changes in the cerebellum following developmental exposure to ethanol. Alcohol Alcohol Suppl 1993; 2: 199-202

40. Wierzba-Bobrowicz T, Lewandowska E, Kosno-Kruszewska E, Lechowicz W, Skórzewska A, Gwiazda E, Pasennik E. Dendritic and microglial cells in pups of alcohol-treated female rats. Folia Neuropathol 2003; 41: 131-137.

41. Wierzba-Bobrowicz T, Lewandowska E, Stępień T, Szpak GM. Differential expression of calbindin D28k, calretinin and parvalbumin in the cerebellum of pups of ethanol-treated female rats. Folia Neuropathol 2011; 49: 47-55.

42. Zeng Y-S, Xu ZC. Co-existence of necrosis and apoptosis in rat hippocampus following transient forebrain ischemia. Neuroscience Research 2000; 37: 113-125. 